\title{
Precancerous Lesion of the Oral Cavity
}

\author{
Alan J. Drinnan M. B., Ch. B., D.D.S., F.D.S.R.C.S.
}

\section{Introduction}

Although the emphasis in this lecture will be on PRECANCER, it is thought important to review initially some of the critical facts about oral cancer as these features have a bearing on how we regard, recognize and manage precancerous lesions. From the clinical viewpoint, I shall review the major risk factors, systemic and local, which are thought to play a role in the development of precancer and cancer and I shall spend some time on one risk factor which I have had the opportunity to study in some detail and which is well recognized as a serious health problem in the country involved-betel nut chewing in Papua New Guinea.

I shall then review the clinical appearances of some of the oral lesions we believe to be have pre malignant. After this, I shall review a little some of the histological changes and other markers which might help the practitioner separate the high risk lesions from the less worrisome conditions. I shall say something about the clinical diagnostic tests currently available and finally mention some of the treatment modalities which have been proposed for managing pre-malignant lesions. I shall conclude with a look into the future and speculate a little on the outlook for managing precancer. If there is time, I would like to say a word or two about the acquired immunodeficiency syndrome (AIDS) as it affects the mouth. Although the oral conditions seen in AIDS patients are not necessarily believed to be "precancerous lesions", there is still much we do NOT know about AIDS and the mouth. The disease is spreading quickly in South East Asia (although not necessarily so fast in Japan), and should be considered a very serious health problem.

\section{Incidence of Oral Cancer}

Oral cancer, although not a common disease, is of great importance because of the morbidity and mortality associated with it. The incidence of oral cancer varies from a low incidence of one to four percent of all malignancies in Japan, the United States and Western Europe to over 45 percent in parts of $\mathrm{Asia}^{1)}$. Current trends show that there may be a reduction in the incidence of the disease in Asia which may be attributable to changes in the habits of smoking. It has been suggested that, as the incidence of smoking "sophisticated" tobacco products such as manufactured cigarettes increases and the use of crude tobacco smoked with additives goes down, the overall incidence of oral cancer is declining. On the other hand it has been noted that, as the rise in

Dr. Drinnan is Distinquished Service Professor, School of Dental Medicine, State University of New York at Buffalo, Buffalo, New York 14214 U.S.A.

Presented at the 13 th Annual Meeting of Japan Society for Oral Tumors Osaka, Japan, January 27 th, 1995. 
use of tobacco by teenagers in the West increases, the incidence of oral cancer in young people also increases.

One of the difficulties of comparing oral cancer surveys from different countries is that the category "oral cancer" may vary from one in which oral and lip cancers are considered to surveys in which "oral cancer" is considered an all inclusive term which includes lip, intraoral and pharyngeal cancers. It should be understood that the figures cited in this paper ${ }^{1)}$ are believed to represent a general consensus understanding of the epidemiology of oral cancer. It is important to remember that a distinction must be made between the behavior of intraoral cancer and cancers occurring on the lip. Intraoral cancer carries a much greater morbidity and mortality rate than cancers of the lip and generally the figures quoted by Binnie in $1972^{2)}$ remain appropriate today and reflect the relative seriousness of intraoral versus lip cancers. The crude five-year survival rate for lip cancers is over 80 percent, the rate for intraoral cancer is of the order of 30-40 percent. Understandably, survival rates would be expected to vary considerably from country to country depending on the availability and sophistication of medical care.

\section{Factors Influencing Prognosis of Oral Cancer}

Several factors are known to influence the survival rate of intraoral cancer and these include the following:

1. LOCATION: The further back in the mouth, the worse the prognosis.

2. SIZE: The larger the lesion at diagnosis - the worse the prognosis.

\section{CELLULAR DIFFERENTIATION :}

The less differentiated-the worse the prognosis.

4. REGIONAL NODE INVOLVEMENT: The more nodes involved - the worse the prognosis.

\section{DISTANT BLOOD METASTASIS :}

When present-a very poor prognosis.

\section{Epidemiological Factors}

From an epidemiological point of view, the major features of oral cancer include :

1. AGE: The prevalence of the condition increases with age although this may be modified by other factors, such as tobacco use. For example, young teenagers who use tobacco (snuff and chewing tobacco) may develop oral cancer at an earlier age.

2. SEX: The condition is more common in males than in females. This is presumed to be because males are more likely to indulge in known risk habits such as tobacco and alcohol use. In those areas, where women indulge in risk habits at about the same level as men, e.g. chewing tobacco and smoking, the incidence rates of oral cancer are more or less the same between the sexes.

3. ETHNIC FACTORS : These are most probably related to social and cultural practices such as alcohol use, smoking and chewing tobacco, "reverse smoking", chewing betel nut with various additives and so on. Sometimes a "habit" may be carried by immigrants into a "low risk" country and the resultant adoption of the habit result in a higher incidence of oral cancer in that country.

Regional practices also influence the location of an oral cancer. For example, in Western countries, intraoral cancer usually affects the tongue, floor of mouth, buccal mucosa, mandibular alveolar tissues, retromolar region and soft palate. The hard palate and dorsum of the tongue are the sites of lowest incidence. In Southeast Asia the buccal mucosa and commissural mucosae are the most common sites.

\section{Risk Factors Predisposing to Oral Cancer Development}

Oral cancer risk factors are well recognized 
and include :

(1) Tobacco

(2) Alcohol

(3) The use of the areca nut and various additives

(4) Nutritional factors (particularly iron deficiency)

(5) Fungal and viral agents

(6) Physical factors

(7) Immunodeficiency factors

Syphilitic glossitis is now relatively rare, but students were expected to remember it as they had to know the five SSSSS which were thought to predispose to oral cancer.

They were :

1. Spirits (alcohol)

2. Smoking (tobacco)

3. Sepsis (poor oral hygiene)

4. Sex (Males higher incidence than females) and

\section{Syphilis !}

The use of tobacco is well recognized as a major source of intraoral carcinogens. Tobacco may be used alone as in cigars, cigarettes, pipe tobacco or chewed or used as snuff. It may be used in combination with other agents such as in the "betel quid". The betel quid is widely used in Asia and its composition varies from country to country ${ }^{3)}$. Typically, it consists of tobacco flakes, areca nut, lime, catechu and betel vine leaf. Various spices may be added. The precise use of the areca nut varies widely, for example, in Papua New Guinea, the areca nut is chewed on its own and then lime is smeared on the oral mucosa to "recharge" the quid and permit the aromatic alkaloid stimulants to be released. Tobacco is rarely added in Papua New Guinea. In India, tobacco is nearly always part of the quid. The spread of oral cancer from the coastal areas of Papua New Guinea into the highlands is believed to have been directly related to the trading of the coastal areca nuts and lime to the highlanders who, before the development of good roads linking the coast to the highlands, were generally unfamiliar with the habit.

It is not clear whether the chewing of areca nut alone without tobacco increases the risk of developing oral cancer, but there is little doubt that the habit may provoke the development of oral submucous fibrosis which many believed may be considered of premalignant potential. There is, as yet, no clarification of this point and the matter is still being investigated.

Although there is still some dispute about whether alcohol itself is carcinogenic, there is general agreement that its use potentiates the effects of other carcinogens especially those arising from tobacco use. The relatively high incidence of oral cancer in certain parts of France and in Denmark is attributed to the high prevalence of heavy drinking and heavy smoking.

Certain nutritional deficiencies are associated with atrophy of oral mucous membranes and this may make these tissues more susceptible to carcinogenic action. The Paterson-Kelly (Plummer-Vinson) syndrome, in which an iron deficiency anemia is associated with an increased incidence of pharyngeal cancer, is well recognized. It is also believed that variations in vitamins $\mathrm{A}$ and $\mathrm{C}$ may also be of importance and there is evidence that doses of vitamin $\mathrm{A}$ and similar products may produce remission of oral leukoplakias and offer some protection against malignant change in abnormal oral tissues. (see below under CHEMOPREVENTION)

There is a well recognized association between the fungus candida albicans and oral cancer and premalignant conditions such as leukoplakia, but there is no clear understanding as to whether the fungi should be considered opportunistic organisms developing in an area of neoplasia or whether they are significant 
in the etiology of the neoplastic condition. It is generally believed that dysplastic lesions which show significant candida involvement should be monitored closely as they show a greater risk of malignant change. It has been shown that, under certain circumstances candida albicans may be capable of producing known carcinogens, so a direct etiological role may occur $^{4)}$.

There is little doubt that certain viruses can stimulate epithelial growth and the human papilloma virus (HPV), Epstein-Barr virus (EBV) and the cyto-megalo virus (CMV) certainly have been associated with oral neoplasms. The precise role of viruses in producing oral cancer, however, remain unclear ${ }^{5)}$. Suggestions have been made that the immune deficiency state in AIDS may contribute to over Activity of viruses on the oral mucosae.

Physical factors such as ultraviolet radiation are known to play a role in the development of skin cancers such as basal cell carcinoma, malignant melanoma and it may be that they are important in the etiology of lip cancer, but any possible role in oral cancer is unclear.

Perhaps one of the most interesting developments of the last 15 years has been the increased recognition of oral mucosal changes associated with immune deficiency states particularly those induced by the human immunodeficiency virus-HIV. The relationship is not fully understood, but it would seem reasonable to expect any immunosuppression, whether as a result of a virus infection or induced pharmacologically, might make a tissue more susceptible to carcinogens such as oncogenic viruses.

\section{Clinical Aspects of Pre Cancerous Lesions}

I would now like to turn to the question of precancer which, from a practical point-ofview, often presents a serious management pro- blem for the medical and dental practitioner. There is frequently little difficulty in recognizing the well developed squamous cell carcinoma. Its appearance as a nodular or ulcerated, indurated, inflamed lesion usually presents few problems in diagnosis. The more worrying situation is that in which a patient may present with a condition which, although not actually representing a malignant change, is thought to be "precancerous". It is essential that such patients be managed effectively so that, should oral cancer develop, it can be treated with due dispatch.

\section{Definition}

A precancerous lesion has been defined as "A morphologically altered tissue in which oral cancer is more likely to occur than in its apparently normal counterpart." and a precancerous condition is defined as "A generalized state associated with the significantly increased risk of cancer." (World Health Organization (WHO) Working Group Definitions $1978^{6)}$.

There are certain lesions and conditions which are generally recongnized to be precancerous. Precancerous lesions include those which are white (leukoplakia) or red (erythroplasia or erythroplakia) or a mixture of red and white (speckled leukoplakia). Precancerous conditions include those associated with iron deficiency, lichen planus, syphilis, lupus erythematosus and actinic keratosis.

\section{Leukoplakia}

Leukoplakia has been defined as "A white patch or plaque that cannot be rubbed off and cannot be characterized clinically or histologically as any other disease" (WHO definition 1978). It is not associated with any physical or chemical cultivated agent except the use of tobacco. It should be noted that this definition is of a clinical condition and does not 
reflect on the histological changes which may be seen within a "leukoplakia" lesion. Indeed, white areas may reveal little more than hyperkeratinization with no significant epithelial dysplasia whereas others may show varying degrees of dysplastic change. Various studies have reported the incidence of malignant change occurring in leukoplakia and these changes vary from less than one percent malignant change to approximately ten percent. These differences may reflect differences in the selection of the lesions that were studied. However, it is also known that there is great variation in the risk of oral cancer developing even in severely dysplastic lesions and this factor must taken into consideration. It is reasonable to assume a patient with leukoplakia is at a greater risk of developing cancer than a person without a lesion. Silverman has estimated this risk as seven times more in women and 4.8 times more in men $^{7)}$. Silverman also reported evidence that the early detection and treatment of small lesions of oral cancer or precancer significantly improve the prognosis ${ }^{8)}$. Waldron and Shafer reviewed a large number (3256) of biopsy specimens which had been diagnosed clinically as leukoplakia. They found that over $80 \%$ of the lesions showed hyperorthokeratosis, hyperparakeratosis, or acanthosis without epithelial dysplasia. They estimated about three to six percent of clinical leukoplakias would be carcinomas ${ }^{9}$.

\section{Erythroplakia}

The definition of erythroplakia has the same form as that of leukoplakia. The WHO (1978) definition states it is a "A lesion of the oral mucosa that presents as a bright red velvety plaque which cannot be characterized clinically or pathologically as being due to any other condition."

Shafer and Waldron evaluated a series of red lesions and found that there was a significantly higher percentage of erythroplastic lesions which showed severe cellular dysplasia or frank carcinoma than the leukoplakias reported in their study cited above. Over $90 \%$ of 64 red lesions examined showed severe epithelial dysplasia or CIS or invasive squamous cell carcinoma. They concluded that red lesions were much more likely to have malignant potential than white ones ${ }^{10)}$. The increased malignant potential of red lesions has also been reported by Shear ${ }^{11)}$.

Much has been written about the premalignant potential of oral lichen planus. It is generally agreed that there may be a slightly increased risk of a lichen planus patient eventually developing an oral cancer but the reported incidences of such developments have been very variable. Eisenberg and Krutchkoff have reviewd this subject and discussed the significance of lichenoid dysplasia ${ }^{12)}$.

\section{Histological Features of Precancerous Lesions}

From a clinical point-of-view, the clinician must remember that any lesion whether white, red or a "mixed" color could be malignant and clearly it is necessary for the histological features of these lesions to be evaluated. It is generally agreed that the more severe the level of dysplasia, the more likely the lesion is to become malignant. There have been several systems of determining dysplasia and these are generally based on changes of maturation of the epithelial cells and evidence of disturbed cell proliferation. Many pathologists classify the dysplasias into mild, moderate, or severe using the term carcinoma in situ (CIS) as the most severe dysplastic change short of carcinoma. Many believe that in CIS the malignant transformation has indeed occurred, but invasion is not yet demonstrable. There is not always an agreement among pathologists as to the degree of dysplasia shown in a particular 
lesion as there is variation in the usage of the terms mild, moderate and severe dysplasia. These disagreements sometimes may make comparative studies between investigators difficult. The author normally uses the term CIS to characterize the most severe dysplasia and understands it to infer dysplastic changes extend through the full thickness of the epithelium. Smith and Pindborg ${ }^{13)}$ developed a photographic atlas of varying degrees of dysplasia to be used for histological grading of oral epithelial atypia and this has proven very useful as a reference tool. Unfortunately, it is now out of publication. In 1984, Pindborg and colleagues conducted a survey of the histological grading of photographs of dysplastic epithelium ${ }^{14)}$. A total of 72 interested individuals participated in this exercise (not all of them oral pathologists) and each was asked to grade a series of 9 photographs of dysplasia into one of 7 categories ranging from "No dysplasia" through "mild" or "moderate" or "severe" dysplasia up to "frank invasive squamous cell carcinoma". It was determined that there was no uniformity in the gradings. The authors noted that the results of the survey gave reasons... "to worry: that a patient's lesion by one pathologist may be called mild dysplasia and by another frank squamous cell carcinoma".

Several attempts have been made to develop grading systems for evaluating the sysplasias of precancer.

Anneroth and colleagues ${ }^{15-17)}$ developed a scheme that considered four features of the epithelium along with three features of the host-neoplasm relationship. Subsequent modifications have been made to this scheme, but it is now recommended that six features should be evaluated ( 3 features of the epithelium and 3 of the neoplasm-host interaction) and these be quantified by giving a score of one to four each of the six features. The total then gives an indication of the "premalignancy" rating.
Anneroth and colleagues suggested assessing the epithelium as to

1. degree of keratinization

2. nuclear pleomorphism

3. mitoses above the basal cell layer

The highest score-four for each featurewould go to the lesion with

1. no keratinization

2. showing a marked degree of nuclear pleomorphism and

3. many mitoses above the basal layer.

The neoplasm-host interactions would apply only to an already malignant neoplastic oral cancer, but any epithelial features in a noninvasive lesion might be of great importance.

In summary, it can be stated that:

1. Leukoplakia is less likely to show severe dysplastic changes than erythroplasia or speckled erythroplasia and

2. The more severe the degree of dysplasia, the greater chance of the lesion becoming malignant.

\section{Clinical Diagnostic Tests}

The more usual diagnostic aids which the clinician uses to assist in the diagnosis of oral cancer and precancer consist of biopsy, exfoliative cytology and the technique of toluidine staining of tissue. Biopsy, whether excisional or incisional, is thought to need no elaboration in this paper. Exfoliative cytology is generally considered a poor way to establish a tissue diagnosis. There may be very special circumstances in which a conventional biopsy may not be possible but, even though a cytologic smear can harvest dysplastic cells from an oral lesion, it is much less reliable than a biopsy specimen and few surgeons would be prepared to operate on a person without having the benefit of an histologic diagnosis.

The use of the dye toluidine blue has been "in" and "out" of fashion for some years. Some workers, who have had considerable ex- 
perience and skill in using the technique, advocate its use ${ }^{18)}$. However many feel that, unless the user has much experience, the technique may not be easily managed or interpreted. The principle of the test is that certain dysplastic and neoplastic cells take up more of the metachromatic dye than the surrounding normal cells. This feature will allow any stained dysplastic or neoplastic epithelium to "stand out" and permit the clinician to determine the extent of any change. The technique consists of

1. Cleaning the mucosa to be examined

2. A pplying a $1 \%$ toluidine blue dye to the mucosa for about one minute

3. Decolourising the stained area with $1 \%$ acetic acid and then examining any residual stained areas. It cannot be assumed that ALL stained areas are neoplastic or dysplastic as they may represent inflamed areas. If there is such a possibility it is recommended that the test be repeated after 2 weeks at which time it could usually be assumed that any inflammatory condition would have resolved and any repeat staining may represent dysplastic epithelium. Toluidine blue kits are presently being marketed in some countries (for example, Canada) and concerns have been expressed as it is believed that the dye might have some mutagenic effects. Several Canadian oral pathologists have recommended that the dye test not be promoted until any possible deleterious effects have been fully investigated. ${ }^{19)}$

More recently photodynamic therapy (PDT) has been reported by several groups ${ }^{20-22)}$. A photosensitizing substance is given to the patient and is selectively retained by any neoplastic or dysplastic cells. Subsequent treatment of the affected tissues by laser causes destruction of the sensitized cells but does not effect the normal cells surrounding. This technique offers great possibilities and is being actively studied.

\section{Chemoprevention}

Numerous studies have been reported on the effectiveness of treating "pre cancerous" lesions, particularly leukoplakias, with antioxidant supplements such as Vitamin A (retinol), retinoids (natural or synthetic analogues of retinol), beta-carotene and alpha-tocopherol (active form of vitamin $\mathrm{E})^{23)}$.

Some success has been reported in reducing or reversing the keratinization seen in some leukoplakias but the side effects of the vitamin A supplements (13-cis-RA and tretinoin) have been a problem and in one study $79 \%$ of the patients had side effects attributed to the medications $^{24)}$.

The subject has been reviewed by Kaugars and $\mathrm{al}^{25)}$ and they also report on a study in which patients with hyperkeratosis or epithelial dysplasia were treated for 9 months with beta-carotene, alpha tocopherol and ascorbic acid. They observed an over $55 \%$ improvement in the patients, especially in those who reduced their intake of alcohol and tobacco use. There was some benefit noted even in those who did not reduce the "risk factors". The authors concluded "the benefit of antioxidant supplementation in the treatment of oral leukoplakia is uncertain but evidence suggests that it does play a role".

\section{Summary and Outlook}

It is clear that the problem of oral cancer and precancer remains a mystery. Much research utilizing the latest techniques of molecular biology is being conducted and there is every hope that there will be significant advances in our understanding of the process of "pre cancer".

A recent review article is recommended as it summarizes the quest for finding a reliable marker of the premalignant state. Johnson and his colleagues ${ }^{26}$ reviewed the main areas 
of current research which include investigations into

1. Histopathology features

2. Biochemical profiles

3. Abnormalities of cell proliferation

4. Ploidy and karyotyping

5. Micronuclei in buccal smears

6. Markers of Immune function

7. Oncogenes and anti-oncogenes

8. Viral involvement

9. DNA adducts

10. Growth factors and cytokines.

The authors provide 214 references and the interested reader is referred to that their paper for further information. They conclude optimistically :

"the explosion of knowledge of cancer cell biology and of modern molecular biology and its technology will result in rapid progress in the near future, particularly in understanding the series of genetic events cumulatively necessary and sufficient for malignant transformation of oropharyngeal epithelia".

In the interim, the clinician must be sure that he/she understands the latest developments in the field both clinical and "basic science" and should continue to exercise high quality, informed, clinical judgment in managing those patients at risk for oral cancer.

\section{REFERENCES}

1) Johnson N. W. (Editor): Risk Markers for Oral Diseases. Volume 2 ORAL CANCER Detection of Patients and Lesions at Risk. Cambridge University Press, 1991.

2) Binnie H. H., Cawson R. A., et al.: Oral Cancer in England and Wales. Series SMPS No. 23. London, HMSO, 1972.

3) Daftary D. K., Murti P. R., et al. : Risk factors and risk markers for oral cancer in high in. cidence areas of the world. Chapter 2 in ORAL CANCER op cit (Ref. 1)

4) Krogh P., Hald B. and Holmstrup P.: Possible mycological etiology of oral mucosal cancer: catalytic potential of infection with Candida albicans and other yeasts in production of N-nitrosobenzylmethylamine. Carcinogensis $8: 287-288$,
1987.

5) Scully C.: Viruses and oral squamous carcinoma. Oral Oncology, European Journal of Cancer 28 B : 57-59, 1992.

6) World Health Organization Working Group Report: Definitions of leukoplakia and related lesions: An aid to studies of oral precancer. Oral Surgery, Oral Medicine, Oral Pathology 46 : 518539, 1978.

7) Silverman S., Bhargava K., et al. ' Malignant transformation and natural history of oral leukoplakia in 57, 518 industrial workers in Gujarat, India. Cancer 38: 1790-1795, 1976.

8) Silverman S. : Early diagnosis of oral cancer Cancer 62 : 1796-1799, 1988.

9) Waldron C. and Shafer W. : Leukoplakia revisited: a clinicopathological study of 3,256 oral leukoplakias. Cancer 36 : 1386-1392, 1975.

10) Shafer W. and Waldron C. : Erythroplakia of the oral cavity Cancer 36:1021-1028, 1975.

11) Shear M.: Erythroplakia of the mouth International Dental Journal $22: 460-473,1972$.

12) Eisenberg E. and Krutchkoff, D. J. : Lichenoid lesions of oral mucosa, Diagnostic criteria and their importance in the alleged relationship to oral cancer, Oral Surgery, Oral Medicine, Oral Pathology 73 : 699-704, 1992.

13) Smith C. and Pindborg J. J. : Histological Grading of Oral Epitheial Atypia by the Use of Photographic Standards. 1969 Copenhagen Bogtrykkeri.

14) Pindborg J. J., Reibel J. and Holmstrup P.: Subjectivity in evaluating oral epithelial dysplasia, carcinoma in situ and initial carcinoma. Journal of Oral Pathology $14: 698-708,1985$.

15) Anneroth G., Hansen. and Silverman S.: Malignancy grading in oral squamous cell carcinoma Journal of Oral Pathology 15 : 162-168, 1986.

16) Anneroth G., Batsakis J. and Luna M. : Malignancy grading of squamous cell carcinoma in the floor of the mouth related to clinical evaluation. Scandinavian Journal of Dental Research 1986 : 347-358.

17) Anneroth G., Batsakis J. and Luna M. : Review of the literature and a recommended system of malignancy grading in oral squamous cell carcinomas. Scandinavian Journal of Dental Research $95: 229-249,1987$.

18) Mashberg A. and Samit A.: Early Detection, Diagnosis, and Management of Oropharyngeal Cancer. CA-A Cancer Journal for Clinicians 39 : $2: 67-88,1989$.

19) Awde J. D., Bentley K. C., et al. : Oral Cancer Diagnosis. Letter to the Editor Journal of the Canadian Dental Association 60:262, 1994.

20) Schweitzer V. G.: Photodynamic therapy for treatment of head and neck cancer. (Review) 
Otolaryngology-Head and Neck Surgery 102(3) : 225-232, 1990.

21) Mang T. S., McGinnis C., et al. : Fluorescence detection of tumors: studies on the early diagnosis of microscopic lesions in preclinical and clinical studies. Journal of Cell Pharmacology 3: 132-140, 1993.

22) Grant W. E., Hopper C., et al. : Photodynamic therapy of oral cancer: photosensitisation with systemic aminolaevullnic acid. The Lancet 342 : 147-148, 1993.

23) Garewal S. H. and Meyskens F. Jr. : Retinoids and Carotenoids in the Prevention of Oral Cancer: A Critical Appraisal. Cancer Epidemiology, Biomarkers and Prevention 1: 155-159, 1992.

24) Hong W. K., Endicott J. and Itri L. M. : 13-
cis-Retinoic acid in the treatment of oral leukoplakia. New England Journal of Medicine 514 : 1501-1505, 1986.

25) Kougars G. E., Silverman S., et al. : A Review of the Use of Antioxidant Supplements in the Treatment of Human Oral Leukoplakia. Journal of Cellular Biochemistry, Supplement 17 F, 292298, 1993.

26) Johnson N. W., Ranasinghe A. W. R. and Warnakulasuriya K. A. A. S.: Potentially malignant lesions and conditions of the mouth and oropharynx: natural history-cellular and molecular markers of risk. European Journal of Cancer Prevention, Supplement 2, 2 : 31-51, 1993.

本論文の要旨は，第13回日本口腔腫瘍学会総会（平成 7 年 1 月，大阪）において特別講演された。 